\title{
ON AN IDENTITY OF BLOCK AND MARSCHAK ${ }^{1}$
}

\author{
BY J. G. VAN DER CORPUT
}

Communicated by Edwin Moise, July 29, 1959

In the Bulletin of the American Mathematical Society ${ }^{2}$ H. D. Block and Jacob Marschak proved for each choice of the positive integers $m$ and $n$ with $m \leqq n$ the identity

$$
\begin{aligned}
& \sum_{1}\left\{\left(u_{s_{1}}+u_{s_{2}}+\cdots+u_{s_{n}}\right)\left(u_{s_{2}}+u_{s_{3}}+\cdots+u_{s_{n}}\right) \cdots\right. \\
& \quad=\left\{\left(u_{1}+u_{2}+\cdots+u_{m}\right) u_{2} u_{3} \cdots u_{n}\right\}^{-1},
\end{aligned}
$$

where $u_{1}, \cdots, u_{n}$ denote indefinite numbers and where $\sum_{1}$ is extended over all the permutations $\left(s_{1}, s_{2}, \cdots, s_{n}\right)$ of $(1,2, \cdots, n)$ which rank 1 before each of the numbers $2,3, \cdots, m$.

In this paper I shall prove: If $p, q$ and $n$ denote integers with $0 \leqq p \leqq q \leqq n$ and $n \geqq 1$, then

$$
\begin{aligned}
& \sum_{2}\left\{\left(u_{s_{1}}+u_{s_{2}}+\cdots+u_{s_{n}}\right)\left(u_{s_{2}}+u_{s_{8}}+\cdots+u_{s_{n}}\right) \cdots\right. \\
& =\left\{\left(u_{1}+u_{2}+\cdots+u_{q}\right)\left(u_{2}+\cdots+u_{q}\right)\right. \\
& \begin{aligned}
& \cdots \\
& \left.\left(u_{p}+\cdots+u_{s_{n}}\right) u_{s_{n}}\right\}^{-1}
\end{aligned}
\end{aligned}
$$

where $\sum_{2}$ is extended over the permutations $\left(s_{1}, s_{2}, \cdots, s_{n}\right)$ of $(1,2, \cdots, n)$ which rank 1 before $2 ; 2$ before $3 ; \cdots ; p-1$ before $p$ and finally $p$ before each of the numbers $p+1, p+2, \cdots, q$.

The particular case $p=1, q=m$ yields (1).

In the proof of (2) I treat first the case $q=n$. Then $\sum_{2}$ is extended over the permutations $\left(s_{1}, \cdots, s_{n}\right)$ with $s_{h}=h(1 \leqq h \leqq p)$, where $\left(s_{p+1}, \cdots, s_{n}\right)$ is an arbitrary permutation of $(p+1, \cdots, n)$. In this case we must show that

$$
\sum_{2}=\left\{\left(u_{1}+\cdots+u_{n}\right)\left(u_{2}+\cdots+u_{n}\right) \cdots\right.
$$

$$
\left.\left(u_{p}+\cdots+u_{n}\right) u_{p+1} \cdots u_{n}\right\}^{-1} .
$$

In the case $p=n$ the sum $\sum_{2}$ consists of only one term namely

1 This paper is sponsored by the United States Army under Contract No. DA-11022-ORD-2059.

2 H. D. Block and Jacob Marschak, An identity in arithmetic, Bull. Amer. Math. Soc. vol. 65 (1959) pp. 123-124. Without loss of generality we may choose $i=1$ in their identity and then this identity assumes the simpler form indicated in (1). 
$\left(u_{1}+\cdots+u_{n}\right)^{-1}$. I may therefore assume that $p$ is $\leqq n-1$ and that (3) has already been proved with $p$ replaced by $p+1$. For each integer $h \geqq p+1$ and $\leqq n$ the contribution to $\sum_{2}$ of the permutations $\left(s_{p+1}, \cdots, s_{n}\right)$ with $s_{p+1}=h$ is according to the induction hypothesis equal to

$u_{h}\left\{\left(u_{1}+\cdots+u_{n}\right)\left(u_{2}+\cdots+u_{n}\right) \cdots\right.$

so that

$$
\left.\left(u_{p+1}+\cdots+u_{n}\right) u_{p+1} \cdots u_{n}\right\}^{-1}
$$

$\sum_{2}=\left\{\left(u_{1}+\cdots+u_{n}\right)\left(u_{2}+\cdots+u_{n}\right) \cdots\right.$

$$
\left.\left(u_{p+1}+\cdots+u_{n}\right) u_{p+1} \cdots u_{n}\right\}^{-1} \sum_{h=p+1}^{n} u_{h},
$$

which gives the required result (3).

Finally we treat the case $p \leqq q \leqq n-1$ and we may assume that (2) has already been proved with $p$ replaced by $p+1$. We must prove that

$$
u_{p+1} u_{p+2} \cdots u_{n} \sum_{2}=[p+1, p+2, \cdots, q] /[1,2, \cdots, q],
$$

where

$$
\begin{array}{r}
{\left[a_{1}, a_{2}, \cdots, a_{t}\right]=\left(u_{a_{1}}+u_{a_{2}}+\cdots+u_{a_{t}}\right)\left(u_{a_{3}}+u_{a_{3}}+\cdots+u_{a_{t}}\right)} \\
\cdots\left(u_{a_{t-1}}+u_{a_{t}}\right) u_{a_{t}} ;
\end{array}
$$

the right hand side means 1 if $t=0$.

By the induction hypothesis the contribution to $u_{p+1} \cdots u_{n} \sum_{2}$ of the permutations $\left(s_{1}, s_{2}, \cdots, s_{n}\right)$ which rank $p$ before $q+1$ is equal to $[p+1, \cdots, q+1] /[1, \cdots, q+1]$; the contribution to $u_{p+1} \cdots u_{n} \sum_{2}$ of the permutations which rank $q+1$ between $h-1$ and $h$ is for each integer $h$ with $2 \leqq h \leqq p$ equal to

$$
u_{q+1}[p+1, \cdots, q] /[1, \cdots, h-1, q+1, h, \cdots, q]
$$

and finally the contribution to $u_{p+1} \cdots u_{n} \sum_{2}$ of the permutations which rank $q+1$ before 1 is equal to

$$
u_{q+1}[p+1, \cdots, q] /[q+1,1, \cdots q] .
$$

In this way we find

$$
\begin{aligned}
u_{p+1} u_{p+2} \cdots u_{n} \sum_{2}= & \frac{[p+1, \cdots, q+1]}{[1, \cdots, q+1]} \\
& +u_{q+1} \sum_{h=1}^{p} \frac{[p+1, \cdots, q]}{[1, \cdots, h-1, q+1, h, \cdots, q]} .
\end{aligned}
$$


It is therefore sufficient to prove that

$$
\begin{array}{r}
\frac{[p+1, \cdots, q+1]}{[1, \cdots, q+1]}+u_{q+1} \sum_{h=1}^{p} \frac{[p+1, \cdots, q]}{[1, \cdots, h-1, q+1, h, \cdots, q]} \\
=\frac{[p+1, \cdots, q]}{[1, \cdots, q]} .
\end{array}
$$

This identity is obvious for $p=0$, so that I may assume that $p$ is $\geqq 1$ and that (5) has already been proved with $p$ replaced by $p-1$.

The term with $h=1$ occurring on the left hand side of (5) is equal to $\left(u_{1}+\cdots+u_{q+1}\right)^{-1}$ times

$$
u_{q+1} \frac{[p+1, \cdots, q]}{\left(u_{1}+\cdots+u_{q}\right)[2, \cdots, q]},
$$

so that this term is a rational function of $u_{1}$ which possesses at $u_{1}=-\left(u_{2}+\cdots+u_{q+1}\right)$ a simple pole with residue

$$
-[p+1, \cdots, q] /[2, \cdots, q]
$$

and at $u_{1}=-\left(u_{2}+\cdots+u_{q}\right)$ a simple pole with residue

$$
[p+1, \cdots, q] /[2, \cdots, q] \text {. }
$$

The left hand side of (5) is therefore a rational function of $u_{1}$ which possesses at $u_{1}=-\left(u_{2}+\cdots+u_{q+1}\right)$ a simple pole with residue

$$
\begin{array}{r}
\frac{[b+1, \cdots, q+1]}{[2, \cdots, q+1]}+u_{q+1} \sum_{h=2}^{p} \frac{[p+1, \cdots, q]}{[2, \cdots, h-1, q+1, h, \cdots, q]} \\
-\frac{[p+1, \cdots, q]}{[2, \cdots, q]} .
\end{array}
$$

This expression assumes, if we replace $u_{2}, u_{3}, \cdots, u_{q+1}$ by $u_{1}, u_{2}, \cdots, u_{q}$, the form

$$
\begin{aligned}
\frac{[p, \cdots, q]}{[1, \cdots, q]}+u_{q} \sum_{h=1}^{p-1} \frac{[p, \cdots, q-1]}{[1, \cdots, h-1, q, h, \cdots, q-1]} & \\
-\frac{[p, \cdots, q-1]}{[1, \cdots, q-1]} &
\end{aligned}
$$

which is equal to zero according to formula (5) applied with $p$ and $q$ replaced by $p-1$ and $q-1$. Consequently the left hand side of (5) is a rational function of $u_{1}$ which has at $u_{1}=-\left(u_{2}+\cdots+u_{q+1}\right)$ a simple pole with residue 0 , so that this function is analytic at that 
point. This function has at $u_{1}=-\left(u_{2}+\cdots+u_{q}\right)$ a simple pole with residue $[p+1, \cdots, q] /[2, \cdots, q]$ and this is also the case with the function occurring on the right hand side of (5). All the terms occurring in (5) are analytic functions of $u_{1}$, apart of the points $u_{1}$ $=-\left(u_{2}+\cdots+u_{q+1}\right)$ and $u_{1}=-\left(u_{2}+\cdots+u_{q}\right)$, so that the difference between the two sides of (5) is a rational function of $u_{1}$ without poles which tends for $u_{1} \rightarrow \infty$ to zero; this difference is therefore identically equal to zero. This completes the proof.

Mathematics Research Center, United States Army, Madison, Wis. 